\title{
Non-radial Pulsations of BA Supergiants and Be Stars
}

\author{
Dietrich Baade \\ European Southern Observatory, Karl-Schwarzschild-Str. 2, \\ D-85748 Garching b. München, Germany
}

\section{Commonalities of Be Stars and BA Supergiants}

If observing time and number of photons are not the limit, it will probably be very difficult to find any Be star or BA supergiant that is not variable. Moreover, there is hardly any major set of observations that is not tempting to explain at least partly in terms of nonradial ( $g$-mode) pulsations. Since a few years ago, such conjectures are also theoretically permissible because improved opacity calculations have established the classical $\kappa$-mechanism as a viable source of pulsation driving (cf. Pamyatnykh, these proceedings).

Contrary to Be stars, it can for any given BA supergiant nevertheless be arbitrarily difficult to diagnose nonradial pulsations (NRP's) with certainty because they need to be detected against considerable background 'noise' of other physical processes, most of which are related to mass loss and/or rotation. To make things worse, there is some evidence that NRP's can have some effect on the dynamics of the mass loss. On the other hand, variable and non-spherical winds is the subject of this Colloquium, and this paper is accordingly biased towards the interplay between pulsation and mass loss.

\section{BA Supergiants}

\subsection{Overview}

Extensive new ground- (e.g., Spoon et al. (1994)) and space-based (e.g., van Leeuwen et al. (1998)) photometry confirms the long-known universality of variabilities in luminous BA stars but puts it on a more systematic basis.

In optical spectroscopy, the tell-tale signatures of nonradial pulsations are at most occasionally detected against the dominating variations due to rotation and mass loss (Kaufer et al. (1997); see also Kaufer, these proceedings). In normal BA supergiants, Kaufer et al. (1997) do not find a dependency of the radial velocity (RV) variations on the depth of formation of the spectral lines. Therefore, the RV variability is due to some global process, as expected for NRP, and not a consequence of the variable wind. This is different in early B-type hypergiants (Rivinius et al. (1997); see also Wolf and Rivinius, these proceedings), where such a depth dependency does exist. However, when the 
wind is unperturbed, the $\mathrm{H} \alpha$ emission strength of these stars is well correlated with the time integral of the RV curve, which probably scales with the change in radius. Therefore, if the RV variations are due to pulsations, the latter would contribute significantly to the mass loss.

In the large majority of luminous BA stars, their periods seem to be confined between the maximal acceptable period of the radial fundamental mode and the shortest possible rotation period, which is the domain covered by nonradial $g$-modes (e.g., Kaufer et al. (1997) and Lamers et al. (1998)). Lamers et al. (1998) find, that the pulsation constants of S Dor star are typically twice as large as in normal BA supergiants, and attribute this to the larger fraction of the initial mass previously lost by the $\mathrm{S}$ Dor stars. They also conclude that the observed periods are an order of magnitude longer than the ones expected for strange modes. However, since virtually all stars show, often major, season-to-season variations of their periods, the identification of the variability with $g$-mode pulsation is still only a statistical one.

\subsection{HD 64760: Nonradial Pulsation and Mass Loss}

In a fine example of maximal extraction of information from data with poor signal-to-noise (S/N) ratio, Howarth et al. (1998) simultaneously derive photospheric and wind periods from a 16-d series of high-resolution IUE spectra. They find an 8.9-hr (or its 1-c/d alias, $14.2 \mathrm{hr}$ ) and a 1.2-d period in the photospheric line profile variability. Remarkably, the broad absorption troughs of the UV wind lines show the same 1.2-d period, at times also its first subharmonic of $2.4 \mathrm{~d}$. Accordingly, the seed of photospheric perturbations by the nonradial pulsation possibly propagates into the high-velocity wind regime.

Because of the low $\mathrm{S} / \mathrm{N}$, the significance of these results is limited. But the agreement with similar variability patterns in $\mathrm{O}$ stars (cf. Henrichs, these proceedings) is convincing. Towards later spectral subclasses comparable findings are probably more difficult to obtain because (i) the preponderance of few NRP modes decreases, (ii) the periods are becoming comparable to the length of an observing season, and (iii) other wind instabilities and rotational modulation gain in importance.

\section{Be Stars}

Until the end of the 1970's the typical time scales sampled by observations of Be stars were weeks to years, and the phenomena covered were the waxing and waning of the circumstellar disks, $V / R$ variations of emission line profiles, and suspected and actual orbital motions. Only in the 1980's was it discovered that Be stars also undergo comparatively more subtle spectroscopic and photometric variations with periods near one day. Henceforth many observational efforts have focussed on properly sampling this newly discovered time scale. 
The at present only spectroscopic series of observations at high resolution and $\mathrm{S} / \mathrm{N}$, that covers both short ( 1 day) and medium (months) time scales, is the one obtained by Rivinius et al. (1998a, b, c, d, e) of $\mu$ Centauri (HR 5193, B2 IV-Ve). Since by virtue of its temporal structure this data set permits much more far-reaching hypotheses to be formulated than any other one currently does, this section intentionally concentrates on only this one star (but due to space limitations merely a very brief account of its extremely complex variability can be given). In Sect. $3.2, \mu$ Cen is then put into a perspective with numerous observations of other Be stars.

\subsection{Pulsation-driven Mass Loss Events in $\mu$ Centauri?}

\section{Multiperiodic line profile variability}

The line profile variability (lpv) of this star can be decomposed into at least 6 periods which could be determined to within nearly one part in 10,000 (Rivinius et al. (1998b)). Two periods are near $0.28 \mathrm{~d}$ whereas the other four cluster around $0.5 \mathrm{~d}$. Furthermore, the latter four show a distinct pattern in that the difference between the two lowest and the two highest frequencies is $0.034 \mathrm{c} / \mathrm{d}$ each whereas the separation of the two middle frequencies is $0.017 \mathrm{c} / \mathrm{d}$. The $\mathrm{lpv}$ is the same within either of the two groups of periods but differs between the groups. A first preliminary analysis (Rivinius 1998, private communication) yields satisfactory fits with NRP velocity fields corresponding to $\ell=2$ and $m=-1$ for the 0.5 -d periods; $\ell=3$ and $m=-1$ is still an acceptable solution but $\ell=1$ appears excluded. The fitting of the 0.28-d periods is still more tentative. But -2 seems to be the best value for $m$, and $\ell$ is probably in the range 3-5 with $\ell=3-4$ reproducing the observations best. Cyclically repeating line-emission outbursts

$\mathrm{Mu}$ Cen undergoes frequent outbursts with a common morphology (Rivinius et al. (1998a); Rivinius et al., these proceedings). Matter is ejected above the stellar surface and possibly partly merges with a pre-existing Keplerian disk (for references concerning the Keplerian or non-Keplerian character of disks of Be stars and their discussion see J. Bjorkman, these proceedings).

The most intruiging discovery (Rivinius et al., 1998c, d, e) is that the temporal spacing between outbursts corresponds to the beat periods of the 0.5 -d modes having the second and third largest amplitude with the strongest mode, i.e. 55.5 and 29.5 days. There seems to be a threshold for outbursts in the combined amplitude of the participating modes: Only these 2 combinations exceed it whereas the sum of the highest and the lowest amplitude as well as the sum of the second and the third largest amplitude are too small. Moreover, only modes with the same surface velocity field, i.e. the same $\ell$ and $m$, and/or very similar periods can contribute to an outburst, because the sum of the respective strongest mode in the $0.28-\mathrm{d}$ and $0.5-\mathrm{d}$ periods exceeds the threshold but outbursts with their beat period of only $\sim 0.6 \mathrm{~d}$ are not observed. This situation is fortunate in so far as a larger number of possible combinations would have put the star into a permanent outburst stage in 
which no temporal regularity would have been detected. The presence of two photospheric beat periods in circumstellar processes doubles the significance of the inferred causal connection between NRP and mass loss events and re-confirms the multiplicity of the 0.5 -d period.

Osaki (these proceedings) has proposed a model in which the superposition of modes leads to so strong non-linearities that the involved waves break. In this case the matter velocity temporarily equals the phase velocity, which for prograde low- $|m|$ modes is larger than the equatorial rotation velocity, and may, therefore, exceed the critical rotational velocity.

\subsection{Supporting Observational Evidence from Other Stars}

With not even one other $\mathrm{Be}$ star known to exhibit the same processes as $\mu$ Cen, their relevance for the Be phenomenon in general cannot even be speculated about. But partial comparisons can be made with other Be stars and entertain the possibility that $\mu$ Cen is not the only star of its kind.

\section{Different NRP patterns in Be and Bn stars}

In addition to the presence or not of emission lines, i.e. a disk, Be and Bn stars, both of which are rapid rotators, also differ in their NRP properties (Smith and Penrod (1985), Baade (1987)): Be stars generally exhibit both low- and higher-order lpv whereas low-order lpv has not so far been detected in very broad-lined $B$ stars without emission lines. A possible general working hypothesis is, therefore, that low-order NRP and the formation of a disk are somehow related as the case of $\mu$ Cen appears to show in more concrete terms.

\section{Outbursts}

The number of reports of outbursts in Be stars is considerable (e.g., Balona (1990), Oudmaijer and Drew (1997)) although they are often left unidentified as such. One of the most interesting results may be hidden in the MACHO database (Cook et al. (1995)). The so-called "bumpers" found in the LMC often seem to be Be stars. Of the two types of light curves, the triangular one with a steeper rise than decline is clearly reminiscent of $\mu$ Cen. But there is also a rounder and more symmetrical type which does not have its correspondence in $\mu$ Cen. This discovery is exciting because of the lower metallicity of the LMC and the metal-dependence of the $\kappa$-mechanism.

Recently, Mennickent et al. (1998) reported photometric outbursts with a period of $470 \mathrm{~d}$ for the proto-typical rapidly variable Be star $\lambda$ Eri. However, if the data (of this publically available set) with phases between 0.9 and 1.1 , which dominate the power spectrum, are plotted versus time, it becomes apparent that they are mainly due to only two events separated by $\sim 470 \mathrm{~d}$. More observations are therefore needed to consolidate this result.

\section{Sensitivity of observational quantities to outbursts}

The above examples show that photometry provides an excellent sensor for outbursts. This may result either from intrinsic variations of the central star that are linked to the outburst physics or from the temporary presence of circumstellar matter or a combination of these two effects. 
By contrast, the total emission strength is a poor tracer of minor outbursts also in other Be stars (Hayes and Guinan (1984), Bjorkman (1994)). Obviously, in most cases the amount of additionally ejected matter is negligible in comparison to the steady-state disk. Only at high spectral resolution and signal-to-noise ratio can variations in the wings of hydrogen emission lines be observed which are due to electron scattering close to the star. The equatorial concentration of these electrons also causes a significant polarimetric signal of the outbursts (Hayes and Guinan (1984), Bjorkman (1994)). The two effects have, in fact, also been observed simultaneously (Baade (1986)).

\section{Competing seasonal and short-periodic variations}

The strong photometric response to outbursts and similar activities implies a significant handicap of photometric techniques in the search for short-term periods, especially if the latter are very closely spaced. The need to correct for a much larger signal than the rapid variability may even discourage observers to attempt a time series analysis (e.g., Pietrzynski (1997)). Even if such a correction is performed very meticulously (e.g., Štefl and Balona (1996)), there is still the question whether the resulting redistribution of the power in the power spectrum makes the detection of multi-periodicity more difficult.

\section{Mysteriously "variable" periods}

One of the enigmas of Be stars is that observers frequently report periods with an accuracy of about $0.1 \%$ for single-season data but are forced to adjust the numbers by about 1\% from season to season (e.g., Stefl and Balona (1996), Sareyan et al. (1998)). This is in marked contrast with $\mu$ Cen in which there is no indication of period variability and the beat periods and phases are reconciled with outbursts several years prior to the lpv observations (Rivinius et al. (1998c), Rivinius et al. (1998d)) and also permit predictions of the future behaviour to be made (Rivinius et al. (1998e)). The recent observations of $o$ And by Sareyan et al. (1998) are of particular interest.

In observing runs from 5 to 11 nights with 6 telescopes at three different longitudes Sareyan et al. (1998) accumulated several thousand photoelectric measurements within 29 days. Typically, the observations spanned 6 hours per night, and with the exception of one observing run all nights seem to have been useful. In spite of this superb sampling, the authors felt compelled to conclude that the primary period of $1.6 \mathrm{~d}$ (plus its first harmonic which leads to a pronounced double-wave light curve) differs by up to $\pm 4 \%$ with formal intrinsic errors of only $1 \%$ or less in different data strings. Moreover, the authors report a correlation between the value of the period and the amplitude of the variation. Over a couple of years, the amplitude can even vary by an order of magnitude and drop to the detection limit.

If this long-term variation is indicative of multi-period beating, the apparent short-term period variations could be due to the same phenomenon: If the 1.6 -d period actually consists of 2 closely spaced periods, the position of the corresponding peak in the power spectrum does not match either of the two real frequencies if it is unresolved. In a light curve with this pseudo-period, 
there will inevitably be a phase drift, leading to the impression of a variable period. On this basis, o And could be the most promising candidate to date for a similar behaviour as found in $\mu$ Cen. However, the confusing effect of additional, transient periods during outburst phases of Be stars (Rivinius et al. 1998a, b; Štefl, these proceedings) must serve as a caveat. Since Sareyan et al. (1998) adjusted the mean differential magnitude of $o$ And to $0.0 \mathrm{mag}$, the actual relevance of this concern cannot be readily estimated.

\section{Conclusions and Perspectives}

It may require quite some time or a fair amount of luck until nonradial pulsations can be convincingly diagnosed in any given luminous BA star. But the collective evidence that there are NRP's in BA supergiants ( $\alpha$ Cygni variables), B hypergiants, S Dor stars, and possibly also $\eta$ Car stars is persuasive. In some cases, an influence of the pulsation on the structure and dynamics of the radiatively driven wind is seen. The most sensitive tracers of this effect are the $\mathrm{H} \alpha$ emission line profile variations and especially the Periodic Absorption Modulations (PAM's) of UV resonance lines (cf. Owocki, these proceedings). Marlborough (1997) has recently compared major outbursts of Be stars to the eruption mechanisms considered for Luminous Blue Variables. Since there are hints that in some Be stars NRP's may contribute to the driving of outbursts, this raises the reverse question whether the eruptions of very luminous BA stars could also be seen in the light of the role that NRP's may play in Be stars.

A weird detail is that in the PAM power spectra of HD 64760 (Howarth et al. (1998)), HD 91969 (Massa's oral presentation of his paper in these proceedings), and $\zeta$ Pup (cf. Berghöfer, these proceedings) strong peaks appear close to one-half of a stellar pulsation frequency. The latitudinal node lines of tesseral modes could lead to such an odd/even effect along the line of sight to the stellar disk. However, the dominance of the first subharmonic of the NRP period also in the X-ray flux of $\zeta$ Pup would argue against such a conjecture.

Among the Be stars, by contrast, bona fide nonradial pulsators are quite readily identified. But only in $\mu$ Cen have so far closely spaced periods been found, that are theoretically expected for high-nonradial order $g$-modes. In this star, the beating of such modes with different $n$ but the same $(\ell, m)$ values, i.e. identical structures of their surface velocity fields, seems to be a key ingredient to the strongly episodic mass loss. Rivinius (private communication) has investigated the question whether the two historical periods around 1918 and 1977-1989, during which persistent $\mathrm{H} \alpha$ emission was not detectable, can be understood in the same context. Although the numerical precision of the four half-day periods is not sufficient for a firm conclusion, it appears possible that at those epochs these four NRP modes were maximally out of phase. However, so long as a second star with such a behaviour has not been found, any generalization is anyway extremely premature. 
Acknowledgement: I thank Stanislav Štefl for helpful comments on the manuscript and Thomas Rivinius for the permission to quote not yet published results of his $\mathrm{PhD}$ thesis work.

\section{References}

Baade, D. (1986): Be Star Newsletter, No. 13, p. 5

Baade, D. (1987): Physics of Be Stars, eds. A. Slettebak and Th.P. Snow, Cambridge Univ. Press, Cambridge, p. 361

Balona, L.A. (1990): MNRAS, 245, 92

Bjorkman, K.S. (1994): Astrophys. Space Science, 221, 335

Cook et al. (1995): Astrophys. Applic. of Stell. Puls., eds. R.S. Stobie and P.A. Whitelock, ASP Conf. Ser., Vol. 83, p. 221

Hayes, D.P., Guinan, E.F. (1984): ApJ, 279, 721

Howarth, I.D., Townsend, R.H.D., Clayton, M.J., Fullerton, A.W., Gies, D.R., Massa, D., Prinja, R.K., Reid, A.H.N. (1998): MNRAS, 296, 949

Kaufer, A., Stahl, O., Wolf, B., Fullerton, A.W., Gäng, Th., Gummersbach, C.A., Jankovics, I., Kovács, J., Mandel, H., Peitz, J., Rivinius, Th., Szeifert, Th. (1997): A\&A, 320, 273

Lamers, H.J.G.L.M., Bastiaanse, M.V., Aerts, C., Spoon, H.W.W. (1998): A\&A, 335,605

Marlborough, J.M. (1997): A\&A, 317, L17

Mennickent, R.E., Sterken, C. Vogt, N. (1998): A\&A, 330, 631

Oudmaijer, R.D., Drew, J.E. (1997): A\&A, 318, 198

Pietrzynski, G. (1997): Acta Astronomica, 47, 211

Rivinius, Th., Stahl, O., Wolf, B., Kaufer, A., Gäng, Th., Gummersbach, C.A., Jankovics, I., Kovács, J., Mandel, H., Peitz, J., Szeifert, Th., Lamers, H.J.G.L.M. (1997): A\&A, 318, 819

Rivinius, Th., Baade, D., Štefl S., Stahl O., Wolf B., Kaufer A. (1998a): A\&A, 333, 125

Rivinius, Th., Baade, D., Štefl S., Stahl O., Wolf B., Kaufer A. (1998b): A\&A, 336, 177

Rivinius, Th., Baade, D., Štefl S., Stahl O., Wolf B., Kaufer A. (1998c): Proceedings of A Half Century of Stellar Pulsation Interpretations: A Tribute to Arthur N. Cox, eds. P.A. Bradley and J.A. Guzik, ASP Conf. Ser., Vol. 135, p. 348

Rivinius, Th., Baade, D., Štefl S., Stahl O., Wolf B., Kaufer A. (1998d): Cyclical Variability in Stellar Winds, eds. L. Kaper and A.W. Fullerton, ESO Astrophys. Symp., ESO, Garching (in press)

Rivinius, Th., Baade, D., Štefl S., Stahl O., Wolf B., Kaufer A. (1998e): Be Star Newsletter, No. 33, p. 15

Sareyan, J.P., Gonzalez-Bedolla, S., Guerrero, G., Chauville, J., Huang, L., Hao, J.X., Guo, Z.H., Adelman, S.J., Briot, D., Alvarez, M. (1998): A\&A, 332, 155

Smith, M.A., Penrod, G.D. (1985): Relat. betw. Chromosph.-coronal Heat. and Mass Loss in Stars, eds. R. Stalio and J.B. Zirker, Osserv. Astron. Trieste, p. 394

Spoon, H.W.W, De Koter, A., Sterken, C. et al. (1994): A\&AS, 106, 141

Štefl, S., Balona, L.A. (1996): A\&A, 309, 787

van Leeuwen, F., van Genderen, A.M., Zegelaar, I. (1998): A\&AS, 128, 117 


\section{Discussion}

S. Shore: I have a question and a comment. Have you looked at any of the magnetic stars, i.e., He-strong stars, that overlap with the $\beta$ Cep range? There could be the sort of trapped modes at the poles that are modulated on the rotational timescales.

As a comment: in the Be stars, the formation of a boundary layer would be expected and this should damp the pulsations. Is there a change in the amplitude of pulsation during outburst?

D. Baade: No, we have not done this. But it is an interesting suggestion. Irrespective of the presence or absence of a disk, the driving of mass loss by pulsation would remove energy from the pulsation. We have not looked for a reduction in amplitude, but given the simultaneous presence of $g$ modes and the short duration of the outbursts proper of only very few days, I suspect that our sampling of these events is insufficient to establish conclusive results.

G. Peters: Have you looked for closely spaced periods around the dominant 0.7-day one in $\lambda$ Eri? Comment 1: I have examined the entire IUE database on $\lambda$ Eri and looked for cyclical wind variability. By using Scargle's method to analyse the equivalent widths of C IV, I find the same 1.3-year period that Mennickent detected in his photometry. Interestingly, most if not all of the variability is in a DAC that appears at $<-1000 \mathrm{~km} / \mathrm{s}$ but slowly shifts to about $-700--800 \mathrm{~km} / \mathrm{s}$ as the outburst progresses. Comment 2: I would like to add that when $\mu$ Cen undergoes an outburst, it can be spectacular, with He I $\lambda 6678$ variability on a time scale of $<1 \mathrm{~m}$ (see forthcoming paper in ApJL, 502, in press).

D. Baade: In the LTPV data of $\lambda$ Eri the 0.7 -day period is just barely detectable. The $\mathrm{S} / \mathrm{N}$ is too low to search for multiple periods around this value. Reply to comment 2: We also find something similar (Rivinius et al. 1998, A\&A 333, 125). 